\title{
The Macquarie-Strasbourg Connection
}

\author{
Agnès Acker ${ }^{\mathrm{A}}$ \\ ${ }^{\text {A } O b s e r v a t o i r e ~ d e ~ S t r a s b o u r g, ~} 11$ rue de l'Université, 67000 Strasbourg, France
}

Received 2009 August 25, accepted 2009 August 25

Planetary nebulae (PN) are powerful tracers of both stellar and Galactic evolution. The capacity of PN to perform these studies is critically dependent on the size of the population, a major problem with a remarkable advance thanks to Quentin Parker and his team, who from 1997 to 2008 discovered an unprecedented sample of $\sim 1250 \mathrm{PN}$ with the deep, high resolution AAO/UKST SuperCosmos Halpha Survey (SHS) of the Southern Galactic Plane (Parker et al. 2005), doubling the sample collected over the previous century, and leading to $\sim 2700$ for the number of known PN today. A highly productive collaboration between Quentin and I has been established since 2001. Our complementary levels of expertise and facilities constitute the Macquarie/AAO/Strasbourg $H-\alpha$ Planetary Nebulae Project.

The new MASH PN were added to the Centre de Données de Strasbourg as a new PN database continuously updated, and detailed in Parker et al. (2006) and Miszalski et al. (2008). In the framework of a cotutelle agreement between the Strasbourg and Macquarie universities, two $\mathrm{PhD}$ projects based on MASH PN have been conducted under the supervision of Quentin and myself, both projects focusing on the mysterious crowded region of the Galactic Bulge. Alan Peyaud proposed new constraints on late stages of stellar evolution and on the dynamics of the
Galactic Bulge (defence 21 December 2005, Strasbourg). Brent Miszalski discovered $\sim 360$ new PN (MASH-II) completing the largest and most representative sample of PN towards the Galactic bulge (defence 15 August 2009). This catalogue offers a unique opportunity to contribute towards two different, largely unexplored research domains: (i) the kinematics of the bulge, and (ii) the role of binary central stars of PN. I may note that it is a wonderful 'Happy End' of my personal research launched with my thesis entitled 'Kinematics, age, and binarity of PN nuclei' (30 years ago).

The happy and friendly collaboration, which links Quentin and I, has been of great benefit to both Strasbourg and Macquarie universities, enabling a more complete exploitation of the new discoveries via the combined mix of expertise in observations, critical data analysis and exploitation via codes and theoretical models. Thank you cher Quentin!

\section{References}

Miszalski, B., Parker, Q. A., Kovacevic, A., Acker, A., Frew, D. J. \& Birkby, J., 2008, MNRAS, 384, 525

Parker, Q. A., Acker, A., Frew, D. J. et al., 2006, MNRAS, 373, 79

Parker, Q. A., Phillipps, S., Pierce, M. J. et al., 2005, MNRAS, 362,689 